\title{
The DEAD-Box RNA Helicase DDX1 Interacts with the Viral Protein 3D and Inhibits Foot-and-Mouth Disease Virus Replication
}

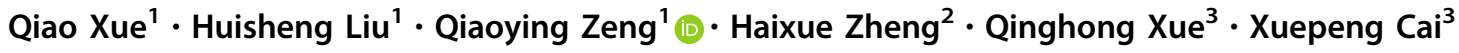

Received: 20 January 2019/ Accepted: 17 May 2019/Published online: 29 July 2019

(c) Wuhan Institute of Virology, CAS 2019

\begin{abstract}
Foot-and-mouth disease virus (FMDV) can infect domestic and wild cloven-hoofed animals. The non-structural protein 3D plays an important role in FMDV replication and pathogenesis. However, the interaction partners of 3D, and the effects of those interactions on FMDV replication, remain incompletely elucidated. In the present study, using the yeast two-hybrid system, we identified a porcine cell protein, DEAD-box RNA helicase 1 (DDX1), which interacted with FMDV 3D. The DDX1-3D interaction was further confirmed by co-immunoprecipitation experiments and an indirect immunofluorescence assay (IFA) in porcine kidney 15 (PK-15) cells. DDX1 was reported to either inhibit or facilitate viral replication and regulate host innate immune responses. However, the roles of DDX1 during FMDV infection remain unclear. Our results revealed that DDX1 inhibited FMDV replication in an ATPase/helicase activity-dependent manner. In addition, DDX1 stimulated IFN- $\beta$ activation in FMDV-infected cells. Together, our results expand the body of knowledge regarding the role of DDX1 in FMDV infection.
\end{abstract}

Keywords Foot-and-mouth disease virus (FMDV) - Interaction · DEAD-box RNA helicase 1 (DDX1) - Antiviral function · Interferon

\section{Introduction}

Foot-and-mouth disease virus (FMDV) is a positive-sense, single-stranded RNA virus that can infect domestic and wild cloven-hoofed animals, including pigs, cattle, sheep, and goats (Belsham 1993; Grubman and Baxt 2004; Robinson et al. 2016). Seven FMDV serotypes have been

Electronic supplementary material The online version of this article (https://doi.org/10.1007/s12250-019-00148-7) contains supplementary material, which is available to authorized users.

Qiaoying Zeng

zengqy@gsau.edu.cn

1 Laboratory of Veterinary Microbiology, College of Veterinary Medicine, Gansu Agricultural University, Lanzhou 730070, China

2 State Key Laboratory of Veterinary Etiological Biology, National Foot and Mouth Diseases Reference Laboratory, Key Laboratory of Animal Virology of Ministry of Agriculture, Lanzhou Veterinary Research Institute, Chinese Academy of Agricultural Sciences, Lanzhou 730046, China

3 China Institute of Veterinary Drug Control, Beijing 100081, China reported (A, O, C, Asia, SAT1, SAT2, and SAT3), which include many subtypes (Carrillo et al. 2005; Dong et al. 2016).

The FMDV genome encodes a single polyprotein that is post-translationally processed into twelve proteins $\left(\mathrm{L}^{\text {pro }}\right.$, VP1, VP2, VP3, VP4, 2A, 2B, 2C, 3A, 3B, 3C ${ }^{\text {pro }}$, and 3D) (Liu et al. 2018). The proteins VP0, VP1, VP3, L ${ }^{\text {pro }}, 2 \mathrm{~B}$, $3 \mathrm{~A}$, and $3 \mathrm{C}^{\text {pro }}$ play roles in inhibiting the host innate immune response (Li et al. 2013, 2016a, b; Zhu et al. 2016; Fan et al. 2017; Rodriguez Pulido and Saiz 2017; Rodriguez Pulido and Sanchez-Aparicio 2018). Additionally, host cell proteins that interact with VP1, 2B, 2C, and 3A have been identified using the yeast two-hybrid system (Gladue et al. 2012, 2014; Liu et al. 2017, 2018). 3D is an important viral protein that has multiple roles in FMDV replication and pathogenesis (Rai et al. 2013; Herod et al. 2016; Rai et al. 2017). An interaction between the host factor Sam68 and 3D has been reported (Rai et al. 2015). To better understand the role of FMDV 3D in viral replication and virulence, we sought to identify new host cell proteins that interact with 3D using the yeast two-hybrid system. 
DEAD-box RNA helicases (DDX) are a family of putative RNA helicases that contain eight conserved amino acid motifs. They play important roles in the regulation of RNA metabolism, including transcription, pre-mRNA processing, RNA decay, RNA export, ribosome biogenesis, and translation (Rocak and Linder 2004). DDX1 is a member of the DEAD-box RNA helicase family, and can inhibit or facilitate viral replication (Edgcomb et al. 2012; Zhou et al. 2017). However, the roles of DDX1 during FMDV infection remain unclear.

In the present study, we used the yeast two-hybrid system, co-immunoprecipitation experiments, and an indirect immunofluorescence assay (IFA) to determine that DDX1 interacted with FMDV 3D. Furthermore, our results demonstrate that DDX1 has anti-FMDV activity. DDX1dependent inhibition of FMDV replication relied, in part, on its ATPase/helicase activity. In addition, DDX1 was involved in FMDV-induced IFN- $\beta$ activation and ISG expression. Collectively, our data expand the body of knowledge regarding the role of DDX1 in FMDV infection.

\section{Materials and Methods}

\section{Cells, Viruses, and Infection}

Porcine kidney 15 (PK-15) cells and human embryonic kidney 293T cells (HEK-293T cells) were maintained in Dulbecco's modified Eagle medium (Thermo Fisher Scientific, Waltham, MA, USA) supplemented with $10 \%$ heatinactivated fetal bovine serum (Gibco) and then maintained at $37{ }^{\circ} \mathrm{C}\left(5 \% \mathrm{CO}_{2}\right)$. The FMDV type $\mathrm{O}$ strain O/BY/CHA/ 2010 was used for viral infections as described previously (Zheng et al. 2013).

\section{Plasmids and Antibodies}

A Myc-tagged expression construct was generated by inserting the cDNA of porcine DDX1 into the pcDNATM3.1/myc-His(-)A vector (Invitrogen, Carlsbad, CA, USA).

The commercial antibodies used in this study include an anti-Myc monoclonal antibody (Santa Cruz Biotechnology, Dallas, TX, USA), an anti-FLAG monoclonal antibody (Santa Cruz Biotechnology), an anti-IRF3 monoclonal antibody (Cell Signaling Technology, Beverly, MA, USA), an anti-P-IRF3 monoclonal antibody (Cell Signaling Technology), an anti-DDX1 polyclonal antibody (Abcam, Cambridge, MA, USA), and an anti- $\beta$-actin monoclonal antibody (Santa Cruz Biotechnology). An anti-VP1 polyclonal antibody was prepared in our laboratory (Li et al. 2017). Anti-3D polyclonal antibody $(500 \mu \mathrm{g} / \mathrm{mL})$ was produced in rabbit by immunization with FMDV 3D protein.

\section{Yeast Two-hybrid Screen}

The yeast two-hybrid screen was performed as described previously (Liu et al. 2017, 2018). Briefly, a porcine cDNA expression library was constructed. GAL4-activation domain-cellular protein fusions were used for identification of interacting proteins. The bait protein, FMDV type $\mathrm{O}$ strain O/BY/CHA/2010 3D, was expressed as an aminoterminal fusion to the GAL4-DNA-binding domain. For screening, the yeast strain expressing the 3D protein was transformed with library plasmid DNA and selected on plates. Once identified, positive library plasmids were sequenced to identify the interacting cellular proteins. DDX1 was recovered from the library and matched the porcine DDX1 sequence (National Center for Biotechnology Information [NCBI] reference sequence GACC01000503.1).

\section{Co-immunoprecipitation and Western Blot Analysis}

PK-15 cells were seeded in 10-cm dishes, and monolayer cells were co-transfected with various plasmids, mock-infected, or infected with FMDV. The cells were then collected and lysed with RIPA buffer, and proteins were immunoprecipitated as described previously ( $\mathrm{Li}$ et al. 2016b). Western blotting was performed as described previously (Zhu et al. 2013). Briefly, the cell samples were lysed with loading buffer. The proteins were analyzed using $12 \%$ SDS-PAGE and transferred onto an Immobilon$\mathrm{P}$ membrane (EMD Millipore, Billerica, MA, USA). The membrane was blocked with $5 \%$ skimmed milk for $2 \mathrm{~h}$, and then incubated with appropriate primary and secondary antibodies. Antibody-antigen complexes were visualized with chemiluminescence detection reagents (Thermo Fisher Scientific, Waltham, MA, USA). The change in abundance of VP1 was determined by densitometric analysis using ImageJ Software and normalized to $\beta$-actin.

\section{Indirect Immunofluorescence Microscopy}

HEK293T cells cultured on Nunc glass bottom dishes (Thermo Fisher Scientific) were transfected with various plasmids using Lipofectamine 3000 (Invitrogen) according to the manufacturer's protocol. At $24 \mathrm{~h}$ post-transfection (hpt), the cells were treated as described previously for detecting the interaction of DDX1 with 3D (Zhu et al. 2016). 


\section{Knockdown of DDX1 Using Small Interfering RNA (siRNA)}

The DDX1 siRNAs were chemically synthesized by GenePharma (Beijing, China). Down-regulation of endogenous DDX1 in PK-15 cells was performed by transfection with DDX1 siRNA using Lipofectamine 2000 (Thermo Fisher Scientific) according to the manufacturer's protocol. A negative control RNA (NC siRNA) was used as negative control. The target sequence for porcine DDX1 is: F: $5^{\prime}-$ GCUUUCAGUAUUCCAGUUATT-3', R: 5'-UAACUGGAAUACUGAAAGCTT-3'.

\section{RNA Extraction and Quantitative Polymerase Chain Reaction (qPCR)}

Total RNAs were extracted from the cell samples with TRIzol reagent (Thermo Fisher Scientific). Moloney murine leukemia virus reverse transcriptase (Promega, Madison, WI, USA) was used to generate cDNAs via reverse transcription (RT)-PCR. SYBR Premix Ex Taq reagents (TaKaRa, Dalian, China) was used to quantify the mRNA level of target proteins in qPCR. The housekeeping gene glyceraldehyde-3-phosphate dehydrogenase $(G A P D H)$ was used as an internal control. Relative mRNA level was calculated with the comparative cycle threshold (CT) $\left(2^{-} \Delta \Delta \mathrm{CT}\right)$ method as described previously (Schmittgen and Livak 2008). The qPCR primers used in this study are listed in Supplementary Table S1.

\section{Statistical Analysis}

The SPSS Statistics for Windows, Version 17.0 (SPSS Inc., Chicago, IL, USA) was used for statistical analysis. Student's $t$ test was used for analyzing three independent experiments. A $* P$ value $<0.05$ was considered significant, and a $* * P$ value $<0.01$ was considered highly significant.

\section{Results}

\section{The FMDV Non-structural Protein 3D Interacts with the Porcine Host Protein DDX1}

The multiple functions of FMDV 3D during viral infection remain unclear. To better understand the role of FMDV 3D in viral replication, we aimed to identify host proteins that interact with 3D using the yeast two-hybrid system. Several host proteins were identified as potential targets of FMDV 3D (Table 1). One of these host proteins, identified as
Table 1 Porcine proteins identified as the potential target of FMDV $3 \mathrm{D}$ by the yeast-two hybrid.

\begin{tabular}{lll}
\hline Gene & Description & Accession number \\
\hline DDX1 & DEAD-box RNA helicase 1 & GACC01000503.1 \\
$V D A C 1$ & Voltage-dependent anion channel 1 & AF268461.1 \\
ATXN3 & Ataxin 3 & NM_001123081.1 \\
\hline
\end{tabular}

porcine host protein DDX1 (NCBI reference sequence GACC01000503.1) was selected for further study.

To confirm the interaction between DDX1 and 3D, PK15 cells were transfected with FLAG-3D expressing plasmid or empty FLAG vector. The cell lysates were immunoprecipitated with anti-DDX1 antibody and analyzed by Western blotting. As shown in Fig. 1A, DDX1 pulled down FLAG-3D. A reverse immunoprecipitation experiment was also performed using anti-FLAG antibody. Similarly, FLAG-3D also immunoprecipitated with DDX1 (Fig. 1B).

To confirm the interaction between DDX1 and 3D in the context of viral infection, PK-15 cells were mock-infected or infected with FMDV at a multiplicity of infection (MOI) of 0.5 . The cell lysates were immunoprecipitated with antiDDX1 antibody. DDX1 pulled down 3D in FMDV-infected cells (Fig. 1C). A reverse immunoprecipitation experiment was subsequently performed using anti-3D antibody, which showed that 3D also immunoprecipitated DDX1 (Fig. 1D). The results confirmed the 3D-DDX1 interaction in the context of viral infection.

The subcellular colocalization of 3D and DDX1 was also examined by IFA. The results indicated an interaction between 3D and DDX1 (Fig. 1E). Taken together, these results confirm that FMDV 3D interacts with DDX1.

\section{FMDV Infection Reduces the Expression of DDX1 Protein}

PK-15 cells were infected or mock-infected with FMDV $(\mathrm{MOI}=0.5)$, and the DDXI mRNA levels and protein abundance were compared at different time points. The expression of DDXI mRNA, viral RNA, and viral titers gradually increased as infection progressed, whereas the abundance of the DDX1 protein was reduced over time (Fig. 2A). Meanwhile, a lower molecular weight band was observed as infection progressed (Fig. 2A).

Expression of DDX1 protein and mRNA remained unchanged in the mock-infected cells (Fig. 2B). Taken together, these results indicate that FMDV infection reduces DDX1 protein expression. 


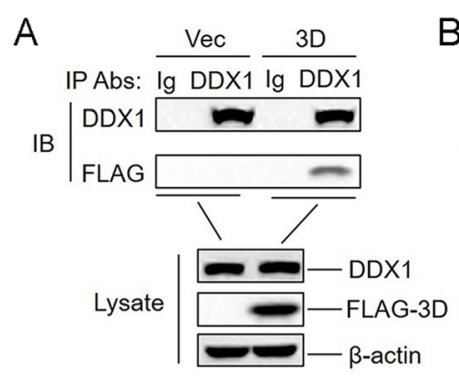

B

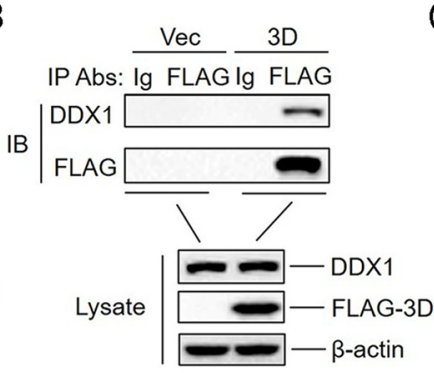

C

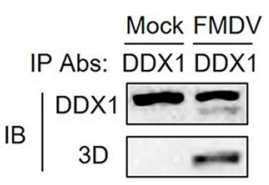

D

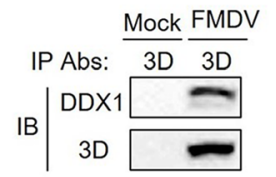

E
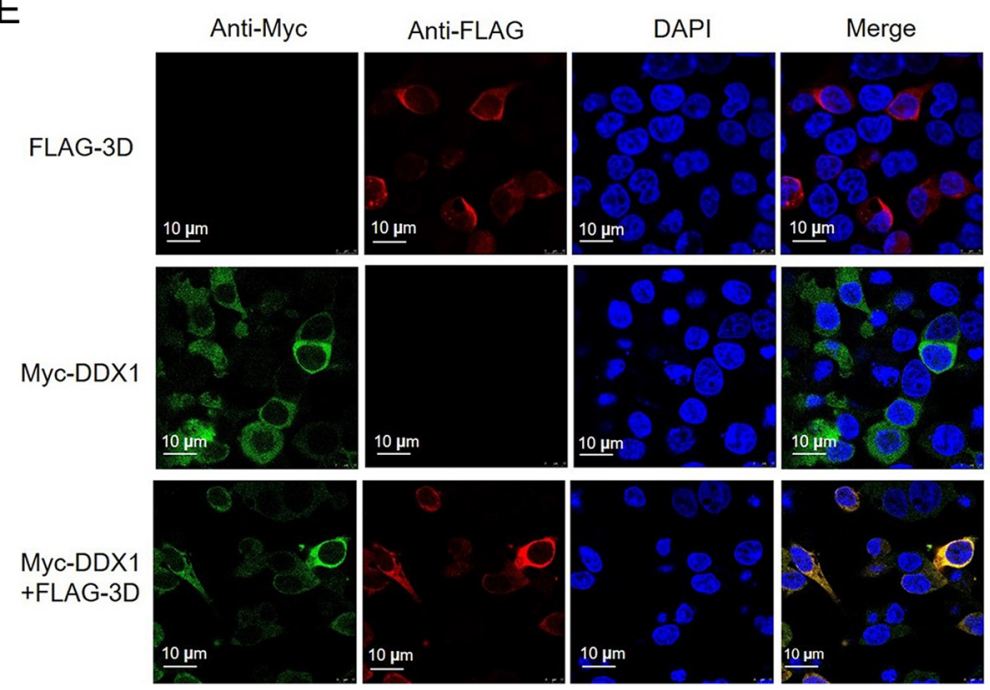

Fig. 1 DDX1 interacts with FMDV 3D protein. A PK-15 cells were seeded in 10-cm dishes, and the monolayer cells were transfected with $10 \mu \mathrm{g}$ FLAG-3D expressing plasmid or $10 \mu \mathrm{g}$ empty FLAG vector. The cells were lysed $32 \mathrm{~h}$ post-transfection (hpt) and cell lysates were immunoprecipitated with anti-DDX1 antibody. The whole-cell lysates and immunoprecipitated antibody-antigen complexes were analyzed by immunoblotting using anti-DDX1 and anti-FLAG antibodies. Ig represents IgG. B Similar infection and immunoprecipitation experiments were performed as described above. However, the lysates were immunoprecipitated with anti-FLAG antibody and analyzed by Western blotting. Ig represents IgG. C PK-15 cells cultured in $10-\mathrm{cm}$ dishes were mock-infected or infected with FMDV $(\mathrm{MOI}=$

\section{DDX1 Inhibits FMDV Replication During Viral Infection}

To assess the roles of DDX1 on FMDV replication, PK-15 cells were transfected with different doses of Myc-DDX1 expressing plasmid. At $24 \mathrm{~h}$ post-transfection (hpt), cells were infected with FMDV (MOI $=0.5)$ for $12 \mathrm{~h}$. As shown in Fig. 3A, the over-expression of DDX1 significantly suppressed FMDV replication in a dose-dependent manner.

FMDV yields were further assessed. PK-15 cells were transfected with DDX1 or NC siRNA for $36 \mathrm{~h}$, and then infected with FMDV $(\mathrm{MOI}=0.5)$. The viral RNA, titers, VP1 protein, and the DDX1 protein were determined at 0 , 6 , and $12 \mathrm{~h}$ after FMDV infection. FMDV replication levels were significantly increased in the DDX1 siRNA-

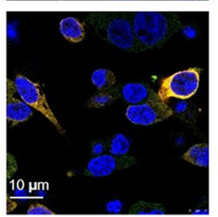

$0.5)$ for $12 \mathrm{~h}$. The cell lysates were immunoprecipitated with antiDDX1 antibody. The antibody-antigen complexes were detected using anti-DDX1 or anti-3D antibodies. D A reverse immunoprecipitation was performed using anti-3D antibody as $(\mathbf{C})$ described. The antibody-antigen complexes were detected by indicated antibodies. E HEK293T cells were cultured in Nunc glass bottom dishes, and the monolayer cells were transfected with $1.5 \mu \mathrm{g}$ FLAG-3D expressing plasmid, $1.5 \mu \mathrm{g}$ Myc-DDX1 expressing plasmid, or $1.5 \mu \mathrm{g}$ empty vector. At $32 \mathrm{hpt}$, the expression of FLAG-3D and Myc-DDX1 was detected by IFA. Cells were double-immunostained for FLAG-3D (red) and Myc-DDX1 (green); cellular nuclei were counterstained with 4',6-diamidino-2-phenylindole (DAPI) (blue).

transfected cells compared with those in the NC siRNAtransfected cells (Fig. 3B, 3C). Taken together, these results demonstrate that DDX1 can inhibit FMDV replication.

\section{DDX1-Dependent Inhibition of FMDV Replication Requires Its ATPase/Helicase Activity}

To analyze whether the ATPase/helicase activity of DDX1 was essential for the inhibition of FMDV replication, a Glu-Gly mutation (DDX1-DGAD) in the conserved DEAD-box motif was generated to abolish the ATPase/ helicase activity, as described previously (Tetsuka et al. 2004; Ishaq et al. 2009). PK-15 cells were transfected with Myc-DDX1 expressing plasmid, Myc-DDX1-DGAD 
Fig. 2 FMDV infection reduces DDX1 protein expression. PK15 cells were seeded in six-well plates and the monolayer cells were infected with FMDV $(\mathrm{MOI}=0.5)(\mathbf{A})$ or mockinfected $(\mathbf{B})$. The cells were collected at $0,4,8,12$, and $16 \mathrm{~h}$. Expression of viral RNA and titers were determined by qPCR and $\mathrm{TCID}_{50}$, respectively (A); expression of DDX1 mRNA and protein were determined by qPCR and Western blotting, respectively $(\mathbf{A}, \mathbf{B}) . * * P<0.01$ versus negative control.

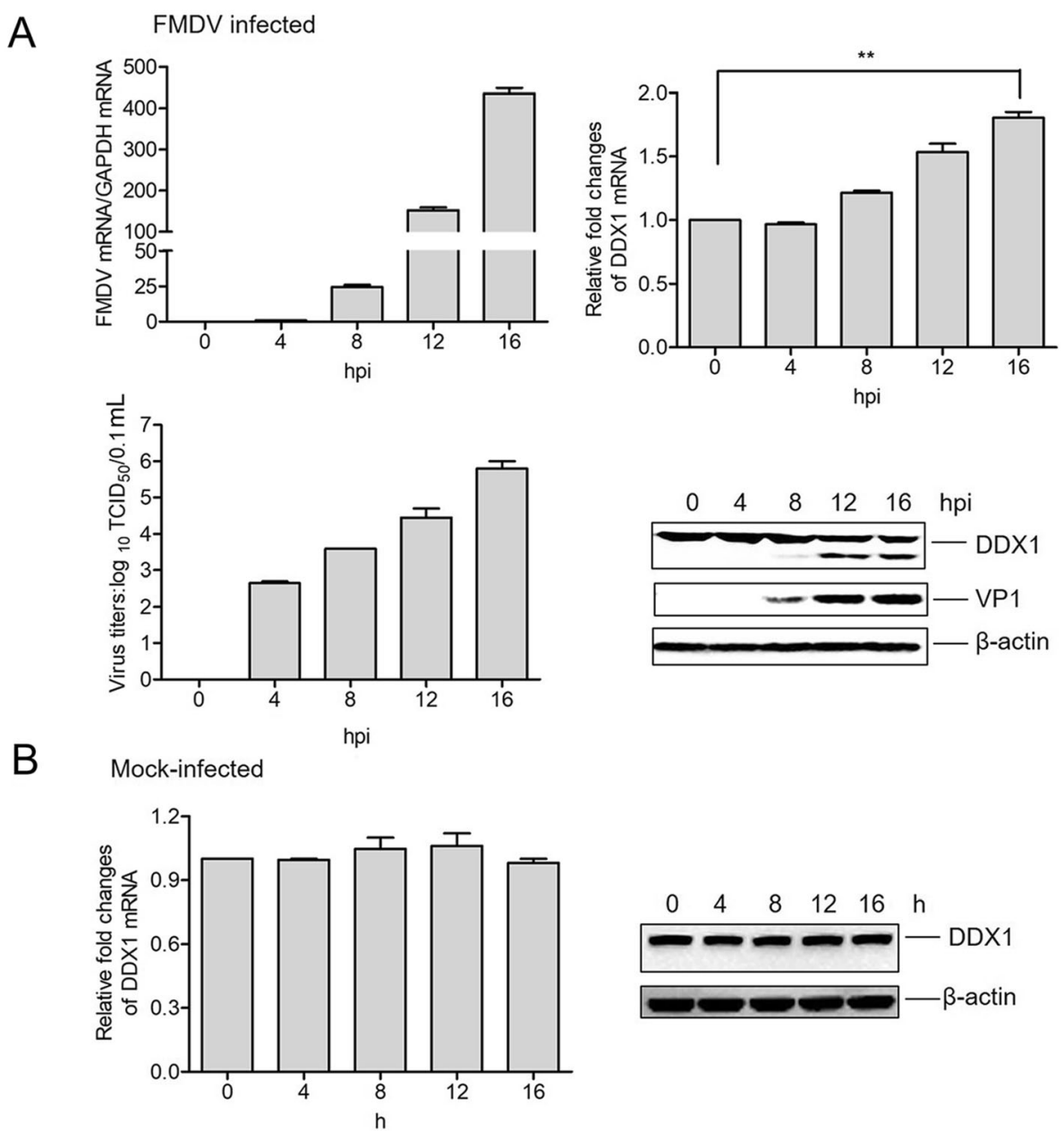

mutant expressing plasmid, or empty vector, and then infected with FMDV (MOI $=0.5)$ for 0,6 and $12 \mathrm{~h}$. Viral RNA, titers, and protein levels were compared. As shown in Fig. 4, the DDX1-DGAD mutant exerted a slight inhibitory effect on FMDV replication, compared to that of wild-type DDX1. Taken together, these results indicate that DDX1-dependent inhibition of FMDV replication might require DDX1 ATPase/helicase activity.

\section{DDX1 is Involved in FMDV-Induced IFN- $\beta$ Activation}

DDX1 can regulate innate immunity (Zhang et al. 2011; Fullam and Schroder 2013; Gu et al. 2017). Thus, we asked whether DDX1 was involved in FMDV-induced IFN- $\beta$ activation. IFN regulatory factor (IRF)-3 phosphorylation is essential for regulating type I IFN gene expression (Tian et al. 2018). Therefore, we examined the impact of DDX1 on the phosphorylation of IRF3 during FMDV infection. IRF3 phosphorylation levels were decreased in DDX1 siRNA-transfected cells, compared with that in NC siRNAtransfected cells (Fig. 3C).
The mRNA levels of IFN- $\beta, O A S 1, I S G 54$, and $M X 1$ were also evaluated by qPCR. This revealed that the mRNA levels of IFN- $\beta, O A S 1, I S G 54$, and MX1 were reduced in $D D X 1$ siRNA-transfected cells compared with those in NC siRNA-transfected cells (Fig. 5). Taken together, these results indicate that DDX1 is involved in FMDV-induced IFN- $\beta$ production.

\section{Discussion}

The interaction between viral and cellular proteins plays important roles in viral virulence. The potential mechanisms by which FMDV proteins interact with host cell proteins are not fully understood. Studies have shown that the host factor, DDX1, can interact with viral proteins and regulate viral replication. For example, the interaction of DDX1 with human immunodeficiency virus (HIV) type 1 Rev protein promotes the viral assembly process (Edgcomb et al. 2012; Lamichhane et al. 2017). Additionally, DDX1 enhances coronavirus replication by interacting with its nonstructural protein 14 (Xu et al. 2010). However, to the best of our 
A

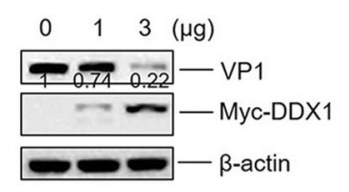

B
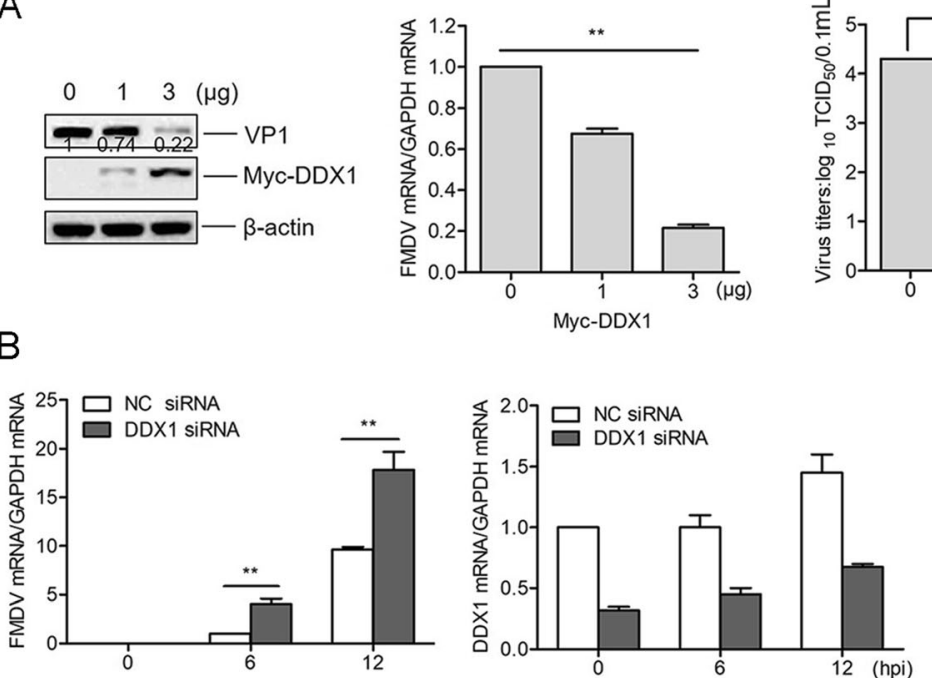

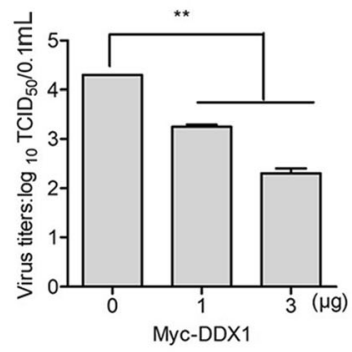

C

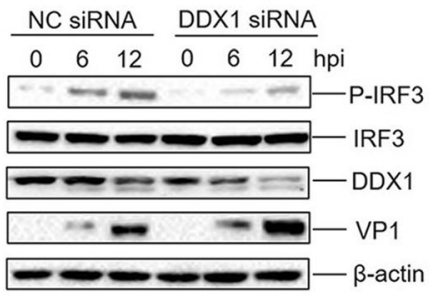

Fig. 3 DDX1 inhibits FMDV replication. A PK-15 cells were seeded in six-well plates and the monolayer cells were transfected with 1 or $3 \mu \mathrm{g}$ Myc-DDX1 expressing plasmid or $3 \mu \mathrm{g}$ empty Myc vector. At $24 \mathrm{hpt}$, the cells were infected with FMDV (MOI $=0.5$ ) for $12 \mathrm{~h}$. Expression of viral VP1 protein was determined by Western blotting and quantified as described; expression of viral RNA was determined by qPCR; viral titers were determined by TCID50. B, C PK-15 cells were seeded in six-well plates and the monolayer cells

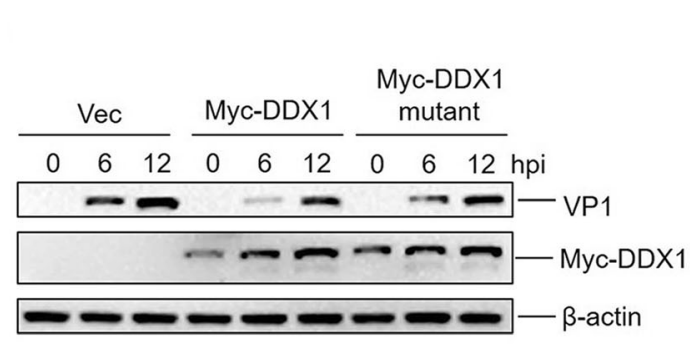

Fig. 4 Inhibition of FMDV replication partly depends on the ATPase/ helicase activity of DDX1. PK-15 cells were seeded in six-well plates and the monolayer cells were transfected with $2 \mu \mathrm{g}$ Myc-DDX1 expressing plasmid, $2 \mu \mathrm{g}$ Myc-DDX1-DGAD mutant expressing plasmid, or $2 \mu \mathrm{g}$ empty Myc vector. At $24 \mathrm{hpt}$, the cells were infected

knowledge, the impact of DDX1 on picornaviruses has not been reported to date. Here, we determined that the FMDV non-structural protein 3D interacted with porcine DDX1. In accordance with this result, we also demonstrated the interaction between 3D and DDX1 during viral infection.

DDX1 facilitates the replication of HIV, human polyomavirus, and coronavirus, whereas it inhibits transmissible gastroenteritis virus (TGEV) replication (Sunden et al. 2007; Zhou et al. 2017). Here, our results suggest that DDX1 inhibits FMDV replication, and that this depends, in part, on its ATPase/helicase activity. Together, these studies reveal the disparate functions of DDX1 during different viral infections. were transfected with $150 \mathrm{nmol} / \mathrm{L}$ nontargeting control (NC) siRNA or DDX1 siRNA for $36 \mathrm{~h}$ followed by infection with FMDV $(\mathrm{MOI}=0.5)$ for 0,6 , and $12 \mathrm{~h}$. Expression of viral RNA and DDX1 mRNA was determined by qPCR assay; the viral titers were determined by TCID50 (B). Expression of DDX1, IRF3, P-IRF3, and viral VP1 proteins were detected by Western blotting $(\mathbf{C})$. ${ }^{* *} P<0.01$ versus negative control.

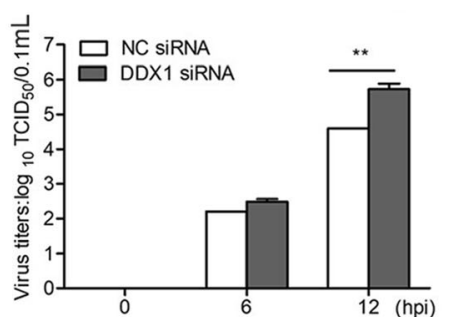

$\square$ Vec

$\square$ Myc-DDX1

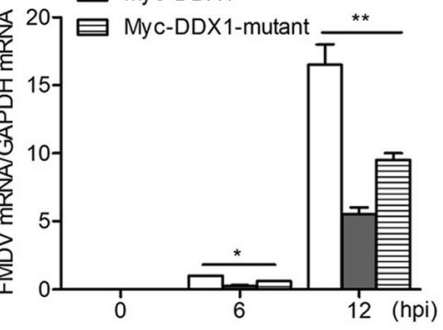

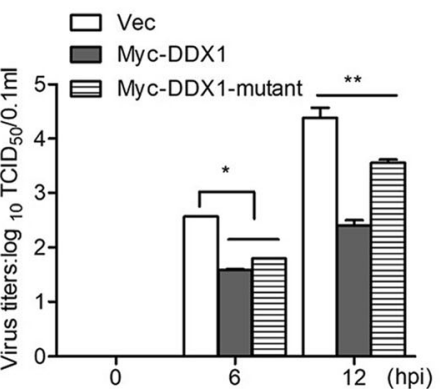

with FMDV $(\mathrm{MOI}=0.5$ ) for 0,6 , and $12 \mathrm{~h}$. Expression of viral VP1 and DDX1 protein was determined by Western blotting; expression of viral RNA was determined by qPCR; viral titers were determined by TCID50. $* P<0.05$ and $* * P<0.01$ versus negative control.

In general, viral infection triggers host innate immune responses through the activation of pattern recognition receptors, resulting in the anti-viral state of an organism (Xue et al. 2018). DDX1, a dsRNA sensor, can activate type I IFN responses through the TRIF pathway (Zhang et al. 2011). Thus, DDX1 may be involved in the activation of innate immune signaling pathways during viral infection. A recent study confirmed that DDX1 is involved in TGEV nsp14-induced IFN- $\beta$ production (Zhou et al. 2017). Based on the results in the present study, we suggest that DDX1 is also involved in FMDV-induced type I IFN production. Notably, both TGEV and FMDV are singlestranded RNA viruses, and further studies are required to 
Fig. 5 DDX1 is involved in FMDV-induced IFN- $\beta$ activation. PK-15 cells were seeded in six-well plates and the monolayer cells were transfected with $150 \mathrm{nmol} / \mathrm{L}$ NC siRNA or DDX1 siRNA for $36 \mathrm{~h}$ followed by infection with equal amounts of FMDV

$(\mathrm{MOI}=0.5)$ for 0,6 , and $12 \mathrm{~h}$.

Expression of IFN- $\beta, I S G 54$,

$O A S 1$, and $M X 1$ mRNA was determined by qPCR assay.

$G A P D H$ was used as an internal control. $* P<0.05$ and $* * P<0.01$ versus negative control.
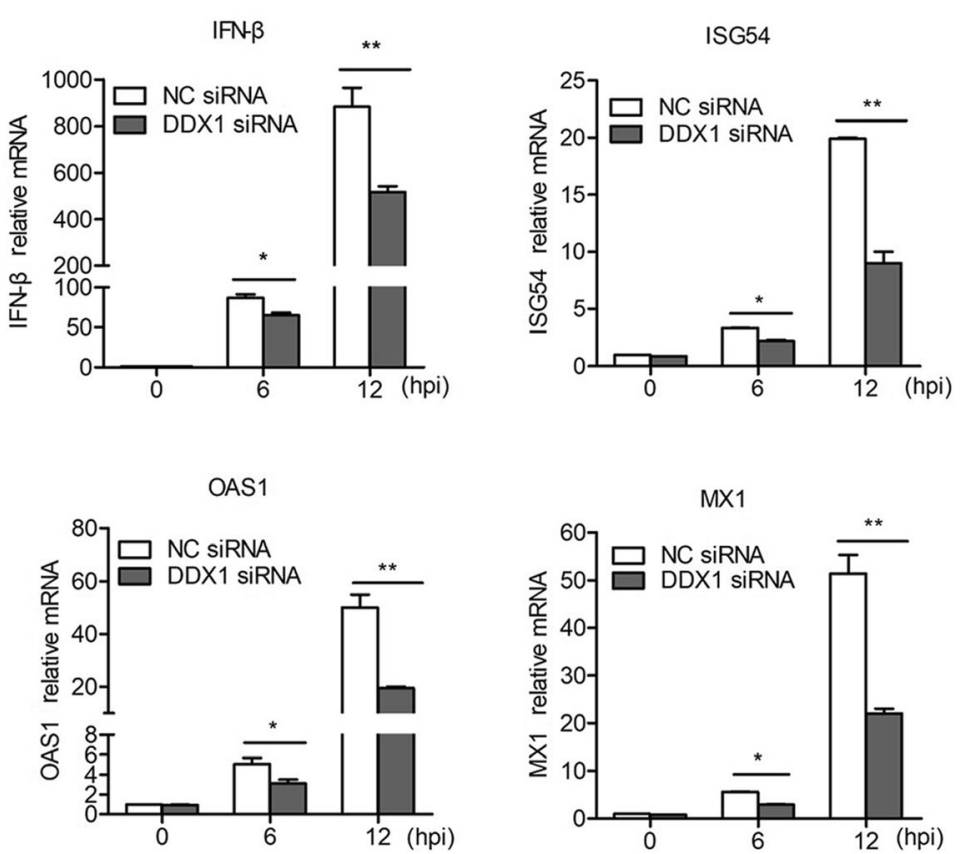

investigate the mechanisms by which DDX1 activates type I IFN during such infections. FMDV is sensitive to type I IFN (Zhu et al. 2016). We therefore infer that DDX1 may inhibit FMDV replication by regulating type I IFN.

Studies have shown that the ATPase/helicase activity is essential for DDX1-mediated innate immune signaling (Ishaq et al. 2009). Therefore, we speculated that the DDX1DGAD mutant would fail to induce type I IFN, thereby compromising its inhibitory effect on FMDV replication. Our results are consistent with DDX1 being a mediator of FMDV-induced type I IFN production. However, the DDX1DGAD mutant suppresses FMDV replication, indicating that DDX1 may exert its anti-FMDV effect through multiple mechanisms. In addition, our results confirmed the interaction between FMDV 3D and the DDX1-DGAD mutant (Supplementary Figure S1), suggesting that the conserved DEAD-box motif is not essential for the 3D-DDX1 interaction. This finding suggests that the 3D-DDX1 interaction may not affect the ATPase/helicase activity of DDX1 or DDX1-induced signaling pathways.

The mechanisms by which FMDV modifies host cell proteins for its own replication, or to evade the host innate immune response, are not fully understood. DDX1 is a multifunctional protein whose expression and function during FMDV infection was previously unknown. Here, we showed that DDX1 expression was reduced during FMDV infection, thus uncovering new roles for DDX1 and providing new insights into the mechanisms by which DDX1 responds to viral infections. As we observed a lower molecular weight band of DDX1 protein after FMDV infection progressed, further studies should be performed to investigate whether FMDV infection induces the cleavage of DDX1.
In conclusion, our results showed that DDX1-dependent inhibition of FMDV replication relied, in part, on its ATPase/ helicase activity. In addition, DDX1 is involved in FMDVinduced type I IFN production. Activating the anti-FMDV functions of DDX1 may provide new strategies to suppress viral replication. Our results also showed that FMDV nonstructural protein 3D interacts with porcine DDX1. However, further studies are needed to investigate the biological significances of the interaction of 3D with DDX1, which may reveal novel pathogenic mechanism mediated by FMDV.

Acknowledgements This study was supported by grants from the National Natural Science Foundation of China (Nos. 31302106, 31260616, and 31602035); and the National Key Research and Development Program of China (Nos. 2016YFD0500901 and 2017YFD0500903).

Author Contributions QZ, XC, and $\mathrm{HZ}$ designed the experiments. QX and HL carried out the experiments. QX, HL, and QHX analyzed the data. QX and HL wrote the paper. All the authors approved the final manuscript.

\section{Compliance with Ethical Standards}

Conflict of interest The authors declare that they have no conflict of interest.

Animal and Human Rights Statement This article does not contain any studies with human or animal subjects performed by any of the authors.

\section{References}

Belsham GJ (1993) Distinctive features of foot-and-mouth disease virus, a member of the picornavirus family; aspects of virus 
protein synthesis, protein processing and structure. Prog Biophys Mol Biol 60:241-260

Carrillo C, Tulman ER, Delhon G, Lu Z, Carreno A, Vagnozzi A, Kutish GF, Rock DL (2005) Comparative genomics of foot-andmouth disease virus. J Virol 79:6487-6504

Dong YM, Cai JC, Chen HT, Chen L (2016) Protection of a novel epitope-RNA VLP double-effective VLP vaccine for foot-andmouth disease. Antivir Res 134:108-116

Edgcomb SP, Carmel AB, Naji S, Ambrus-Aikelin G, Reyes JR, Saphire AC, Gerace L, Williamson JR (2012) DDX1 is an RNAdependent ATPase involved in HIV-1 Rev function and virus replication. J Mol Biol 415:61-74

Fan X, Han S, Yan D, Gao Y (2017) Foot-and-mouth disease virus infection suppresses autophagy and NF-small ka, CyrillicB antiviral responses via degradation of ATG5-ATG12 by $3 \mathrm{C}$ (pro). Cell Death Dis 8:e2561

Fullam A, Schroder M (2013) DExD/H-box RNA helicases as mediators of anti-viral innate immunity and essential host factors for viral replication. Biochim Biophys Acta 1829:854-865

Gladue DP, O'Donnell V, Baker-Branstetter R, Holinka LG, Pacheco JM, Fernandez-Sainz I, Lu Z, Brocchi E, Baxt B, Piccone ME, Rodriguez L, Borca MV (2012) Foot-and-mouth disease virus nonstructural protein $2 \mathrm{C}$ interacts with Beclin 1 , modulating virus replication. J Virol 86:12080-12090

Gladue DP, O’Donnell V, Baker-Bransetter R, Pacheco JM, Holinka LG, Arzt J, Pauszek S, Fernandez-Sainz I, Fletcher P, Brocchi E, Lu Z, Rodriguez LL, Borca MV (2014) Interaction of foot-andmouth disease virus nonstructural protein $3 \mathrm{~A}$ with host protein DCTN3 is important for viral virulence in cattle. J Virol 88:2737-2747

Grubman MJ, Baxt B (2004) Foot-and-mouth disease. Clin Microbiol Rev 17:465-493

Gu L, Fullam A, McCormack N, Hohn Y, Schroder M (2017) DDX3 directly regulates TRAF3 ubiquitination and acts as a scaffold to co-ordinate assembly of signalling complexes downstream from MAVS. Biochem J 474:571-587

Herod MR, Ferrer-Orta C, Loundras EA, Ward JC, Verdaguer N, Rowlands DJ, Stonehouse NJ (2016) Both cis and trans activities of foot-and-mouth disease virus 3D polymerase are essential for viral RNA replication. J Virol 90:6864-6883

Ishaq M, Ma L, Wu X, Mu Y, Pan J, Hu J, Hu T, Fu Q, Guo D (2009) The DEAD-box RNA helicase DDX1 interacts with RelA and enhances nuclear factor kappaB-mediated transcription. J Cell Biochem 106:296-305

Lamichhane R, Hammond JA, Pauszek RF 3rd, Anderson RM, Pedron I, van der Schans E, Williamson JR, Millar DP (2017) A DEAD-box protein acts through RNA to promote HIV-1 RevRRE assembly. Nucleic Acids Res 45:4632-4641

Li X, Wang J, Liu J, Li Z, Wang Y, Xue Y, Li X, Cao H, Zheng SJ (2013) Engagement of soluble resistance-related calcium binding protein (sorcin) with foot-and-mouth disease virus (FMDV) VP1 inhibits type I interferon response in cells. Vet Microbiol 166:35-46

Li D, Lei C, Xu Z, Yang F, Liu H, Zhu Z, Li S, Liu X, Shu H, Zheng H (2016a) Foot-and-mouth disease virus non-structural protein 3A inhibits the interferon-beta signaling pathway. Sci Rep 6:21888

Li D, Yang W, Yang F, Liu H, Zhu Z, Lian K, Lei C, Li S, Liu X, Zheng H, Shu H (2016b) The VP3 structural protein of foot-andmouth disease virus inhibits the IFN-beta signaling pathway. FASEB J 30:1757-1766

Li C, Zhu Z, Du X, Cao W, Yang F, Zhang X, Feng H, Li D, Zhang K, Liu X, Zheng H (2017) Foot-and-mouth disease virus induces lysosomal degradation of host protein kinase PKR by $3 \mathrm{C}$ proteinase to facilitate virus replication. Virology 509:222-231

Liu H, Xue Q, Zeng Q (2017) The kinase STK3 interacts with the viral structural protein VP1 and inhibits foot-and-mouth disease virus replication. Biomed Res Int 2017:2481348
Liu H, Xue Q, Cao W, Yang F, Ma L, Liu W, Zhang K, Liu X, Zhu Z, Zheng H (2018) Foot-and-mouth disease virus nonstructural protein 2B interacts with cyclophilin $\mathrm{A}$, modulating virus replication. FASEB J. https://doi.org/10.1096/fj.201701351:fj201701351

Rai DK, Schafer EA, Singh K, McIntosh MA, Sarafianos SG, Rieder E (2013) Repeated exposure to 5D9, an inhibitor of 3D polymerase, effectively limits the replication of foot-and-mouth disease virus in host cells. Antivir Res 98:380-385

Rai DK, Lawrence P, Kloc A, Schafer E, Rieder E (2015) Analysis of the interaction between host factor Sam68 and viral elements during foot-and-mouth disease virus infections. Virol J 12:224

Rai DK, Diaz-San Segundo F, Campagnola G, Keith A, Schafer EA, Kloc A, de Los Santos T, Peersen O, Rieder E (2017) Attenuation of foot-and-mouth disease virus by engineered viral polymerase fidelity. J Virol 91:e00081-17

Robinson L, Knight-Jones TJ, Charleston B, Rodriguez LL, Gay CG, Sumption KJ, Vosloo W (2016) Global foot-and-mouth disease research update and gap analysis: 5-biotherapeutics and disinfectants. Transbound Emerg Dis 63(Suppl 1):49-55

Rocak S, Linder P (2004) DEAD-box proteins: the driving forces behind RNA metabolism. Nat Rev Mol Cell Biol 5:232-241

Rodriguez Pulido M, Saiz M (2017) Molecular mechanisms of footand-mouth disease virus targeting the host antiviral response. Front Cell Infect Microbiol 7:252

Rodriguez Pulido M, Sanchez-Aparicio MT (2018) Innate immune sensor LGP2 is cleaved by the Leader protease of foot-andmouth disease virus. PLoS Pathog 14:e1007135

Schmittgen TD, Livak KJ (2008) Analyzing real-time PCR data by the comparative $\mathrm{C}(\mathrm{T})$ method. Nat Protoc 3:1101-1108

Sunden Y, Semba S, Suzuki T, Okada Y, Orba Y, Nagashima K, Umemura T, Sawa H (2007) DDX1 promotes proliferation of the JC virus through transactivation of its promoter. Microbiol Immunol 51:339-347

Tetsuka T, Uranishi H, Sanda T, Asamitsu K, Yang JP, Wong-Staal F, Okamoto T (2004) RNA helicase A interacts with nuclear factor kappaB p65 and functions as a transcriptional coactivator. Eur J Biochem 271:3741-3751

Tian J, Liu Y, Liu X, Sun X, Zhang J, Qu L (2018) Feline herpesvirus 1 (FHV-1) US3 blocks type I IFN signal pathway by targeting IRF3 dimerization in a kinase-independent manner. J Virol 92:e00047-18

Xu L, Khadijah S, Fang S, Wang L, Tay FP, Liu DX (2010) The cellular RNA helicase DDX1 interacts with coronavirus nonstructural protein 14 and enhances viral replication. J Virol 84:8571-8583

Xue Q, Liu H, Zhu Z, Yang F, Ma L, Cai X, Xue Q, Zheng H (2018) Seneca Valley Virus 3C(pro) abrogates the IRF3- and IRF7mediated innate immune response by degrading IRF3 and IRF7. Virology 518:1-7

Zhang Z, Kim T, Bao M, Facchinetti V, Jung SY, Ghaffari AA, Qin J, Cheng G, Liu YJ (2011) DDX1, DDX21, and DHX36 helicases form a complex with the adaptor molecule TRIF to sense dsRNA in dendritic cells. Immunity 34:866-878

Zheng H, Guo J, Jin Y, Yang F, He J, Lv L, Zhang K, Wu Q, Liu X, Cai $X$ (2013) Engineering foot-and-mouth disease viruses with improved growth properties for vaccine development. PLoS ONE 8:e55228

Zhou Y, Wu W, Xie L, Wang D, Ke Q, Hou Z, Wu X, Fang Y, Chen H, Xiao S, Fang L (2017) Cellular RNA helicase DDX1 is involved in transmissible gastroenteritis virus nsp14-induced interferon-beta production. Front Immunol 8:940

Zhu Z, Shi Z, Yan W, Wei J, Shao D, Deng X, Wang S, Li B, Tong G, Ma Z (2013) Nonstructural protein 1 of influenza A virus interacts with human guanylate-binding protein 1 to antagonize antiviral activity. PLoS ONE 8:e55920

Zhu Z, Wang G, Yang F, Cao W, Mao R, Du X, Zhang X, Li C, Li D, Zhang K, Shu H, Liu X, Zheng H (2016) Foot-and-mouth disease virus viroporin $2 \mathrm{~B}$ antagonizes RIG-I-mediated antiviral effects by inhibition of its protein expression. J Virol 90:11106-11121 